\title{
Clinical Analysis and Proper Management of the Patients Visited ER Due to Ingested Foreign Body: Single Center Experience
}

\author{
Young Eun Park, M.D., Eun Mee Oh, M.D., Sang Tae Choi, M.D., \\ Jung Nam Lee, M.D., Woon Ki Lee, M.D., Keon Kuk Kim, M.D. \\ Department of Surgery, Gil Medical Center, Gacheon Medicine and Science, Incheon, Korea
}

\begin{abstract}
Purpose: The conventional treatment for ingested foreign bodies (IFB) is removal, which is successful in most cases. However, it can be associated with severe complications, such as gastrointestinal tract perforation, and require emergency surgery. The aim of this study is to analyze clinical data relating to IFB and to develop a proper management plan to reduce the incidence of severe complications.

Methods: Between September 2001 and September 2009, 117 patients visited the emergency room complaining of IFB. Among these patients, 29 were diagnosed with bezoar and were excluded from the study. Medical data for the remaining 88 patients was reviewed retrospectively. For statistical analysis, the foreign bodies (FB) were classified into three subgroups according to their shape (round, sharp, and amorphous).

Results: The median age of patients with IFB was seven
\end{abstract}

\section{INTRODUCTION}

Ingested foreign bodies (IFB), especially those that reach the gastrointestinal (GI) tract, are a common complaint in emergency rooms and sometimes require emergency surgery. It is well known that conservative treatment involving the removal of IFB is mostly successful, with complications such as GI tract perforation presenting in only a few cases (about 1\%)., Children comprise $80 \%$ of the patients needing medical treatment after the ingestion of foreign bodies (FB). IFB can also happen in adults, especially in edentulous, psychiatric, or alcohol-dependent patients. ${ }^{3}$

Depending on the kind of ingested material, patients with IFB present with various symptoms, but asymptomatic patients are also found. In these cases, the diagnosis of IFB can be difficult. ${ }^{4}$ Incidental discovery of IFB can happen during radiological examinations of the abdomen. Some previous reports

Received November 27, 2013, Revised 1st, February 6, 2014;

2nd, February 20, 2014, Accepted February 24, 2014

※ Corresponding author: Sang Tae Choi

Department of Surgery, Gil Medical Center, Gacheon Medicine and Science, 1198, Guwol-dong, Namdong-gu, Incheon 405-760, Korea Tel : +82-32-460-3244, Fax : +82-32-460-3247

E-mail : bcon1218@hanmail.net years, and the male-to-female ratio was 1.3:1. Many of these patients were preschool children under the age of seven who had accidentally swallowed FB (56 cases, 63.6\%). The most common symptom presented among the patients was FB sensation (18 cases, $21 \%$ ). The results of subgroup analysis showed no significant relation between the shape of the FB and the treatment modality. Spontaneous passage was observed in 21 cases $(23.9 \%)$. Otherwise, endoscopic removal was performed successfully in $61.4 \%$ of cases, and 13 patients required emergency operations (14.8\%).

Conclusion: Early diagnosis and a prompt approach are significant in the successful treatment of IFB. Endoscopic or surgical procedures are sometimes required, particularly in cases where complications are suspected.

Key words: Ingested foreign bodies, Complication, Endoscopy show that FB can migrate either to intra-luminal or extra-luminal sites by perforating the GI tract. Perforation by FB through the GI tract causes difficulty in diagnosis, particularly when there is no peritonitis or abscess formation.

Many IFB pass spontaneously through the GI tract without any trouble. Consequently, they are excreted spontaneously or easily removed using endoscopic procedures. In only $1 \%$ of all cases, IFB cause severe complications by perforating the GI tract, resulting in intra-abdominal abscesses or GI tract obstructions. Emergency surgery within the appropriate time cannot be avoided in these cases. ${ }^{5}$

The treatment method for IFB depends on each case and requires consideration of the characteristics, shapes, and location of the FB within the GI tract or abdomen, as well as patients' medical histories and physical examinations. There is no definitive protocol to deal with IFB. Indeed, the treatment can vary depending on the physician's clinical experience. In this paper, we analyze the clinical data of IFB and further attempt to establish a proper management protocol.

\section{MATERIALS AND METHODS}

From September 2001 to September 2009, 117 patients visited the emergency room at Gachon Gil Medical Center due to IFB. The patients' dates of admission were reviewed retro- 
spectively based on electronic medical records. Among them, 29 patients were ultimately diagnosed with bezoar and excluded. The remaining 88 patients were included in the analysis.

In the data analysis, where applicable, the patients' clinical manifestations and underlying disorders, and the initial locations of the IFB, types and characteristics of the IFB, treatment methods, migration of the IFB within the GI tract, perforation of the IFB through the GI tract, and complications were reviewed.

The diagnosis of IFB in the GI tract was confirmed by either endoscopy or plain radiography, which helped to determine the location of the FB and the site of perforation if perforation had occurred. In all patients, plain radiographs, including views of the oropharynx, neck, chest, and abdomen, were performed. Computed tomography (CT) was done in patients with complaints of unusual symptoms, such as right upper quadrant pain and flank pain, and suspected severe complications, such as GI obstructions, perforations, and extra-luminal migration. Surgical interventions were performed in cases of failed endoscopic removal.

IFB were categorized into three subgroups according to the shapes of the IFB. First, hard and circular types of IFB, such as coins or mercury batteries, were named "round type" ( $n=59$, $67 \%$ ). Second, sharp and metallic substances, such as needle and safety pins, were named "sharp type" ( $n=18,20.5 \%)$. Lastly, materials with an irregular shape, such as shells, caps, and pieces of plastic, were distinguished as "amorphous type" $(\mathrm{n}=11,12.5 \%)$. We tried to categorize the IFB by their sizes, however, the cut off value was not obtained statistically.

For the statistical analysis, the student's t-test and Mann-Whitney U-test were used in order to examine the continuous variables, and the chi-square test or Fisher's test were used to analyze the categorical variables. A $p$ value of less than 0.05 was considered statistically significant. The statistical analysis was performed using SPSS version 18.0 (SPSS Inc, Chicago, IL).

The informed consent and approval of the Institutional Review Board were not obtained because of the retrospective nature of this study.

\section{RESULTS}

\section{1) Clinical characteristics of constitutive patients}

The median age of the patients was seven years (range, $1 \sim$ 88 years) and the male-to-female ratio was 1.3:1. Many of the patients were preschool children under the age of seven who had accidentally swallowed FB (56 cases, 63.6\%). Adult patients were found in 27 cases $(30.7 \%)$, and the remaining five cases were school-age children $(5.7 \%)$. Two patients had medical histories of psychiatric disease, one patient was a prisoner, and two patients had a previous history of IFB.

The most common symptom in all 88 patients was FB sensation (27 cases, 31\%), followed by epigastric pain (10 cases, $11 \%$ ) and neck pain (four cases, 5\%).

Following ingestion, the initial location of the FB was in the upper esophagus in 30 cases (34.1\%), middle esophagus in two cases $(2.3 \%)$, lower esophagus in eight cases $(9.1 \%)$, stomach in 34 cases $(38.6 \%)$, small intestine in 11 cases $(12.5 \%)$, and rectum and anus in three cases $(3.4 \%)$.

The median size of the FB was $3 \mathrm{~mm}$ (range, $3 \sim 180 \mathrm{~mm}$ ) and the largest number of IFB was 14 , which were magnets. There was variety in the numbers of FB found in this study.

In the subgroup analysis based on the types of FB, no significant statistical relation was found between the shapes of the FB and the selected treatment modalities $(p=0.110$, data not shown).

2) Clinical progress, management, and complications of constitutive patients

The spontaneous passage of FB was observed in 21 cases (23.9\%).In the majority of other cases, the FB were extracted by flexible endoscopy using forceps (54 cases, 61.4\%), and emergency operations were required in 13 cases (14.8\%). The surgical procedure methods varied according to the location of the FB and the kinds of FB (Table 1).

There were several types of complications in the IFB patients: 10 cases $(11.3 \%)$ presented with impaction in the GI during spontaneous passage without perforation. In two cases (2.2\%), the FB migrated to extra-luminal sites after perforating the GI wall. In one case, flank pain occurred when the FB migrated to the retroperitoneum, while in another case, there were no symptoms. In one case $(1.1 \%)$, the FB migrated to the common bile duct, and the patient complained of fever and chills, which brought ambiguity in the diagnosis.

We also found that additional complications can occur during the removal process. The extraction of multiple, sharp, metallic FB by endoscopy can cause injuries to the GI tract (Fig. 1). Even with small-sized FB, sharp or magnetic materials can cause peritonitis during their natural passage by making directional penetration or fistulous openings inside the intestinal canal. Patients tended to complain of proctodynia because even if the IFB was small, it could impact the anal canal. The sharp or long shape of FB could scrape any site on the GI tract during 
Table 1. Analysis of clinical data of the patients underwent surgical treatment

\begin{tabular}{|c|c|c|c|c|c|c|c|c|}
\hline $\begin{array}{l}\text { Patient } \\
\text { number }\end{array}$ & Sex & $\begin{array}{c}\text { Age at } \\
\text { diagnosis }\end{array}$ & Chief complaint & Type of FB & $\begin{array}{l}\text { Number } \\
\text { of IFB }\end{array}$ & $\begin{array}{c}\text { Migration } \\
\text { or not }\end{array}$ & Location of FB & Surgical treatment method \\
\hline 1 & $\mathrm{~F}$ & 56 & Rt.flank pain & Sewing needle & 1 & Yes & Retropritoneum & Kocher maneuver \\
\hline 2 & M & 28 & Asymtomatic & Sewing needle & 1 & Yes & Lesser omentum & Laparoscopic removal \\
\hline 3 & M & 78 & Low abdominal pain & Fish bone & 1 & Yes & $\mathrm{CBC}$ & Cholecystectomy with T-tube \\
\hline 4 & $\mathrm{~F}$ & 2 & Abdominal pain & Hairpin & 1 & No & Terminal ileum & Segmental resection \\
\hline 5 & $\mathrm{~F}$ & 20 & Abdominal pain & Magnets & 2 & No & G-C/C-C fistula & Wedge resection \\
\hline 6 & $\mathrm{~F}$ & 64 & Low abdominal pain & Fish bone & 1 & No & Rectum & Removal under SA \\
\hline 7 & $\mathrm{~F}$ & 30 & Abdominal pain & Toothbrush & 1 & No & Duodenum & Gastrostomy \\
\hline 8 & M & 40 & Low abdominal pain & Wire,Nail & 14 & No & Stomach & Gastrostomy \\
\hline 9 & M & 4 & Low abdominal pain & Spectacle frame & 1 & No & Stomach & Gastrostomy \\
\hline 10 & M & 5 & Low abdominal pain & Coin & 1 & No & IC valve & Jejunostomy \\
\hline 11 & M & 38 & Low abdominal pain & Animal bone & 1 & No & Rectum & Removal under SA \\
\hline 12 & $\mathrm{~F}$ & 8 & Low abdominal pain & Coin & 1 & No & Stomach & Gastrostomy \\
\hline 13 & M & 48 & Low abdominal pain & Fishing needle & 1 & No & Anus & Removal under SA \\
\hline
\end{tabular}

$\mathrm{G}-\mathrm{C} / \mathrm{C}-\mathrm{C}$ fistula $=$ gastrocolic/coloncolic fistula, $\mathrm{SA}=$ spinal anesthesia.
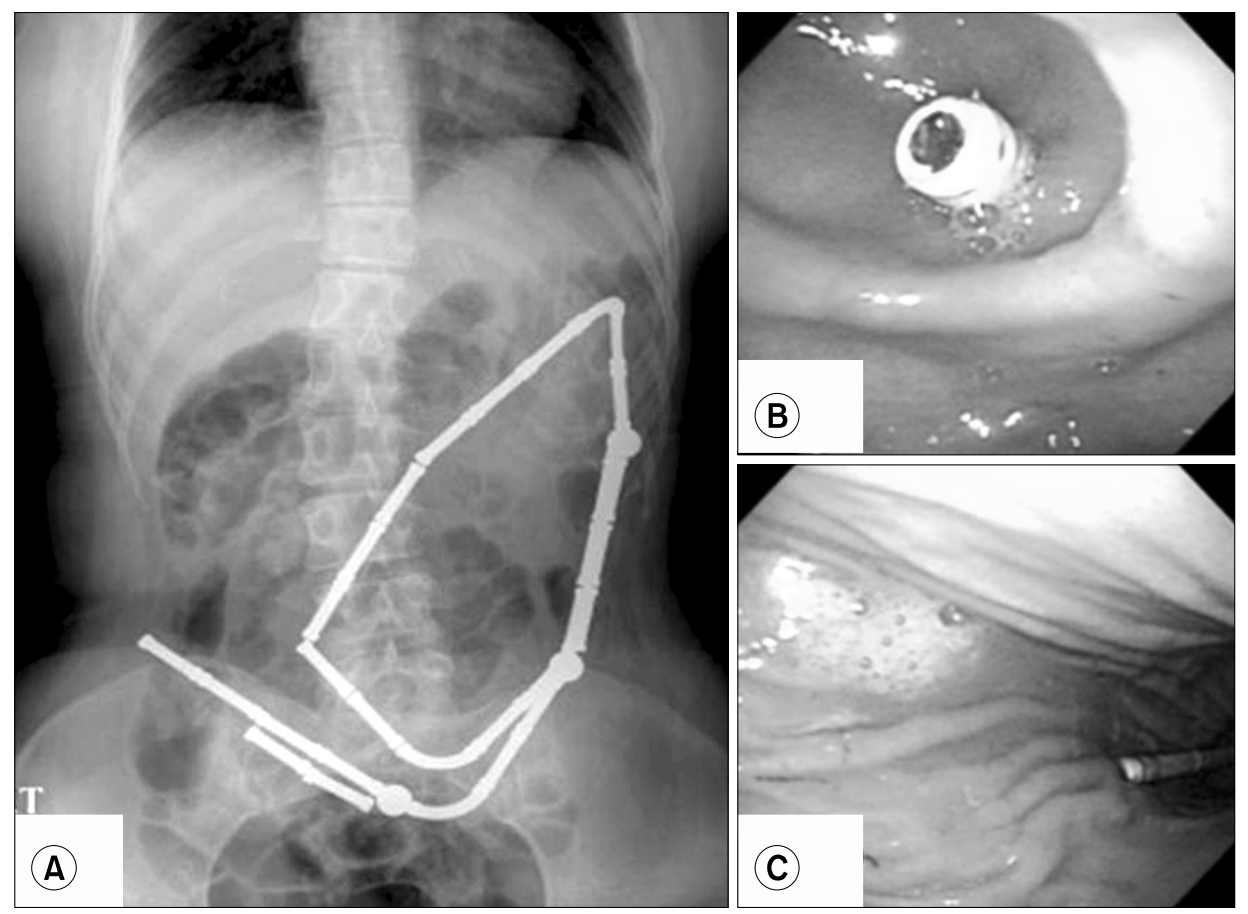

Fig. 1. Simple x-ray shows multiple magnetics are seen at whole abdomen, which suggested the gastro-colic, colo-colic fistula formation. (A) Gastrofibroscopy shows the magnetic is penetrating between proximal antrum and greater curvature of mid-body (B, C).

passage. All the other patients were treated successfully and showed good recoveries without any major complications (Fig. 2).

\section{DISCUSSION}

Due to the mobility and variety of IFB, physicians' clinical experience affected the diagnosis and treatment of IFB which was also dependent on the characteristics of the ingested substance. The aim of this study was to classify the clinical characteristics of IFB and establish a proper management flowchart to deal with IFB.

Previously published studies of IFB have indicated that approximately $52 \sim 97 \%$ of occurrences in pediatric patients are accidental. $^{1,2,6-8}$ These results are similar to the results of this study. The number of IFB patients at preschool age, i.e., between one and seven years old, was 56 , which was $63.6 \%$ of the total study group. In addition, most of the cases revealed 

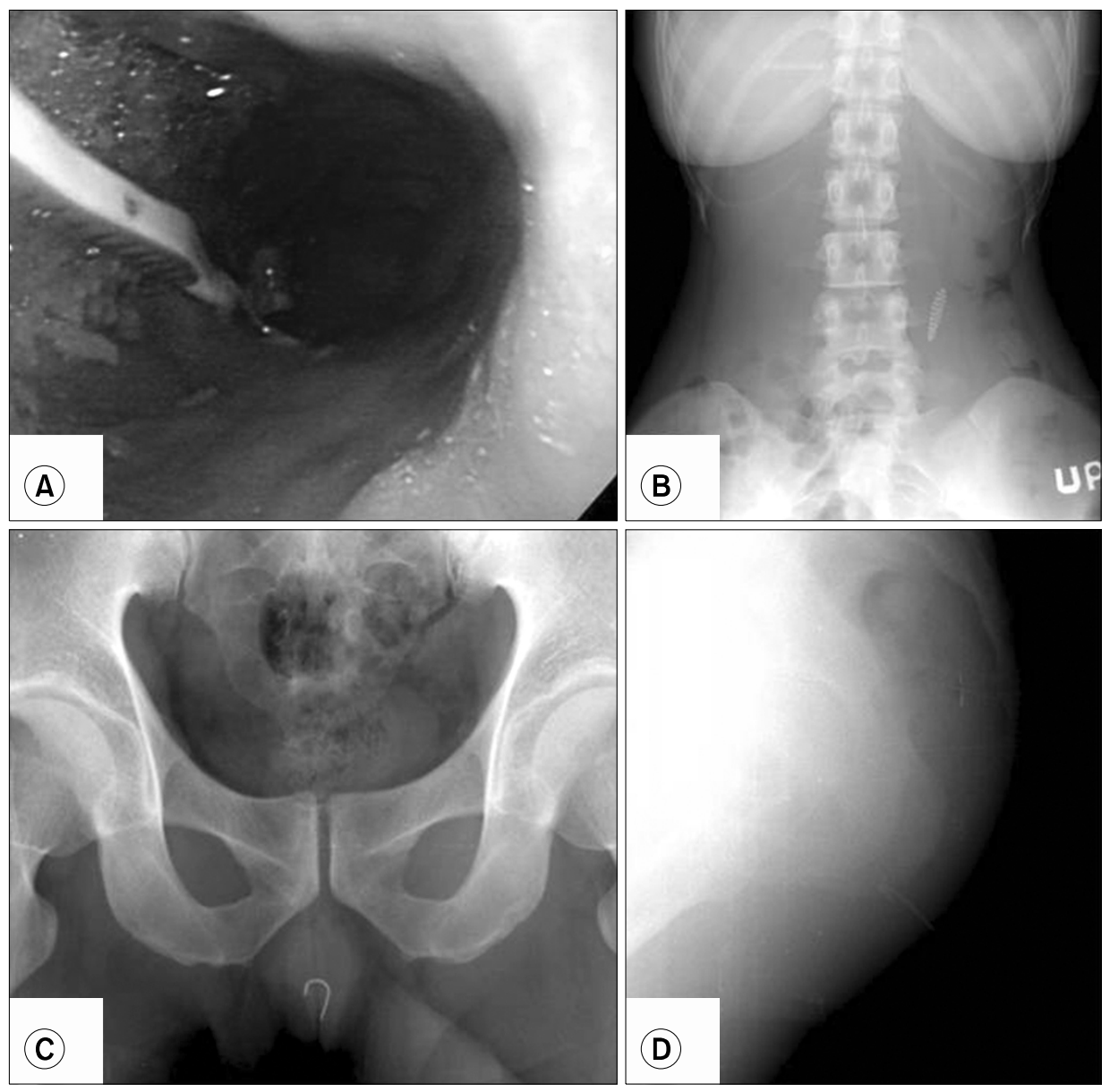

Fig. 2. Gastrofibroscopy shows large type such tooth brush was impacted in stomach (A), simple $\mathrm{x}$-ray shows some radioplaque portion of foreign body (B). Simple x-ray shows sharp metallic type such as needle and pin was impacted in anus during spontaneous passage $(C, D)$.

accidental ingestion. However, in adults, IFB was found to be intentional in the majority of cases, with patients having underlying psychiatric disorders or imprisoned status. ${ }^{3,9-11}$

The type of IFB was associated with the patient's age, social status, and dietary habits. Children often ingested coins and toys during play, while adults usually consumed food materials that were difficult to swallow. The most common types of FB in adults were fish bones and meat., ${ }^{5,12,13}$

The clinical symptoms of IFB varied according to the location of the FB in the patient's body. When FB were located near the pharyngolarynx, the patients usually complained of a feeling of irritation. When FB were located in the upper esophagus, the patients complained of irritation, vomiting, and pain in the pharyngolarynx. For lower esophagus IFB, the patients complained of chest pain, oppressive pain, and ambiguous feelings of irritation. In cases of IFB in the stomach, duodenum, or organs below the duodenum, the patients showed no initial symptoms; however, vomiting, hematemesis, abdominal pain, and fever were shown after IFB removal, leading to complications such as bleeding, perforation, and obstruction. ${ }^{4}$ In this study, most of the patients showed similar symptoms, but unusual symptoms were also observed in some cases. There were several patients who complained of right flank pain, right upper quadrant pain, and anal pain, which was due to the migration of the FB. ${ }^{14}$ These unusual symptoms in IFB should not be overlooked and should be considered important indicators of the possible need for surgical intervention.

FB with sharp ends, such as needles, dental braces, and chicken bones, did not pass easily through the GI, increasing the possibility of complications, like perforation. ${ }^{15-18}$ Indeed, the risk of GI tract perforation can be greater with the increasing degree of sharpness and size of the FB. The sharper and the longer the FB are, the greater their chances of staying in the GI tract, increasing the complication occurrence rate by $15 \sim$ $35 \%{ }^{19}$ Thus, when large irregular-shaped FB are ingested, immediate surgical intervention should be considered rather than waiting for natural excretion or endoscopic removal. We tried to find the relationship between the shape of FB and clinical progress, however there was no significant statistical relation. A greater number of study patients maybe required to obtain 
a statistically significant result.

It was not clear from our data how long FB take to be excreted naturally via the GI tract. However, when FB in the stomach or duodenum are bigger than $6 \mathrm{~cm}$ in diameter, surgical treatment should be undertaken due to the possibility of perforation and bleeding. Even if FB are less than $6 \mathrm{~cm}$ in size, surgical treatment should be considered if the retention time of the FB is longer than $12 \sim 72$ hours. $^{20}$ One case showed the ingestion of a toothbrush, which subsequently impacted the duodenum. A gastrostomy was required in that case.

When surgical treatment is determined for IFB, the general condition of the patient and the location of the FB in the GI tract should be taken into account in order to choose the proper surgical method. FB rarely move to extra-peritoneal spaces or are located in unexpected areas. For that reason, the location of FB must be confirmed before surgery. ${ }^{21}$ If plain radiography does not provide the exact location of the FB, a CT scan might reveal the precise location. Thus, the location of the FB could be an important factor in determining the treatment method. In this study, we found two cases of FB in the retroperitoneum and lesser omentum, and in both cases, surgical treatments were required.

When surgical treatment was selected, generally a laparotomy was performed. Recently however, due to the influence of minimally invasive surgery, laparoscopic surgery has been widely adopted. This cannot only prevent excessive surgical wounds and subsequent complications, such as wound infection and hernia, but can also be beneficial for future surgery in the event of FB re-ingestion. Considering these benefits, a laparoscopic approach should be actively considered for a first IFB event if spontaneous passage and removal using the endoscopic approach have failed.

Sometimes, IFB cannot be found, particularly if they are located within the GI tract or endoperitoneal space. In such cases, if the FB are radiopaque, their location could be discovered with the aid of roentgenoscopy during surgery. Furthermore, if the location of FB in the upper gastrointestinal tract cannot be confirmed, endoscopy could be useful during the operation as it could enhance the visual field and thus confirm the location of the FB within the GI tract. This would help in the efficient removal of the FB from the patient. ${ }^{22,23}$

There were some limitations in this study. First, these results were deduced from a retrospective analysis. Furthermore, the patients' clinical data could have contained missing or incorrect information. However, a prospective study was not possible due to the nature of the condition. Second, selection bias existed in this study due to the small number of patients. Because of these limitations, more satisfactory statistical results were not obtained. For further analysis, large-scale data collection is recommended. Notwithstanding, even given these limitations, we found a fair number of cases showing good examples of proper management and surgical treatment for IFB patients.

\section{CONCLUSION}

In many cases IFB can be removed by either natural excretion or endoscopic retraction. However, when endoscopic removal is impossible or the IFB patient shows unusual symptoms, a different surgical removal approach should be considered depending on the type, size, and shape of the FB.

\section{REFERENCES}

1) Chaves DM, Ishioka S, Felix VN, et al. Removal of a foreign body from the upper gastrointestinal tract with a flexible endoscope: a prospective study. Endoscopy 2004;36:887-892.

2) Kim JK, Kim SS, Kim JI, et al. Management of foreign bodies in the gastrointestinal tract: an analysis of 104 cases in children. Endoscopy 1999;31:302-304.

3) Webb WA. Management of foreign bodies of the upper gastrointestinal tract: update. Gastrointest Endosc 1995;41:39-51.

4) Selivanov V, Sheldon GF, Cello JP, Crass RA. Management of foreign body ingestion. Ann Surg 1984;199:187-191.

5) Goh BK, Chow PK, Quah HM, et al. Perforation of the gastrointestinal tract secondary to ingestion of foreign bodies. World J Surg 2006;30:372-377.

6) Katsinelos P, Kountouras J, Paroutoglou G, et al. Endoscopic techniques and management of foreign body ingestion and food bolus impaction in the upper gastrointestinal tract: a retrospective analysis of 139 cases. J Clin Gastroenterol 2006;40:784-789.

7) Ayantinde AA, Oke AT. A review of gastrointestinal foreign bodies. Int J Clin Pract 2006;60:735-739.

8) Barros JL, Caballero A, Rueda JC, et al. Foreign body ingestion: management of 167 cases. World J Surg 1991;15: 783-788.

9) Blaho KE, Merigian KS, Winbery SL, Park LJ, Cockrell M. Foreign body ingestions in the emergency department: case reports and review of treatment. J Emerg Med 1998;16:21-26.

10) Kamal I, Thompson J, Paquette DM. The hazards of vinyl glove ingestion in the mentally retarded patient with pica: new implications for surgical management. Can J Surg 1999;42: 201-204.

11) Abdullah BJ, Teong LK, Mahadevan J, Jalaludin A. Dental prosthesis ingested and impacted in the esophagus and orolaryngopharynx. J Otolaryngol 1998;27:190-194.

12) Ginsberg GG. Management of ingested foreign objects and 
14 Journal of Minimally Invasive Surgery Vol. 17. No. 1, 2014

food bolus impactions. Gastrointest Endosc 1995;41:33-38.

13) Webb WA. Management of foreign bodies of the upper gastrointestinal tract. Gastroenterology 1988;94:204-216.

14) Choi ST, Lee JN, Kim KK, et al. Laparoscopic removal of an ingested needle, which was penetrated to the lesser omentum of the stomach. J Korean Surg Soc 2006;70:317-320.

15) Theodoropoulou A, Roussomoustakaki M, Michalodimitrakis MN, Kanaki C, Kouroumalis EA. Fatal liver abscess caused by a fish bone. Lancet 2002;359:977.

16) Kumar S, Gupta NM. Foreign bodies migrating from gut to liver. Indian J Gastroenterol 2000;19:42.

17) Losanoff JE, Kjossev KT. Gastrointestinal "crosses": an indication for surgery. J Clin Gastroenterol 2001;33:310-314.

18) Vassilev BN, Kazandziev PK, Losanoff JE, Kjossev KT, Yordanov DE. Esophageal "stars": a sinister foreign body ingestion. South Med J 1997;90:211-214.

19) Vizcarrondo FJ, Brady PG, Nord HJ. Foreign bodies of the upper gastrointestinal tract. Gastrointest Endosc 1983;29: 208-210.

20) Velitchkiv NG, Grigorov GI, Losanoff JE, Kjossev KT. Ingested foreign bodies of the gastrointestinal tract: retrospective analysis of 542 cases. World J Surg 1996;20:1001-1005.

21) Iafrati MD, Fabry SC, Kee YM. A novel approach to the removal of sharp foreign bodies from the stomach using a combined endoscopicand laparoscopic technique. Gastrointest Endosc 1996;43:67-70.

22) Olson JA, Weinstock LB, Brunt LM. Combined endoscopic and laparoscopic approach to remove a sharp gastric foreign body. Gastrointest Endosc 2000;51:500-502.

23) Furihata M, Tagaya N, Furihata T, Kubota K. Laparoscopic removal of an intragastric foreign body with endoscopic assistance. Surg Laparosc Endosc Percutan Tech 2004;14: 234-237. 